\title{
热处理温度对 $\mathrm{LaMgAl}_{11} \mathrm{O}_{19}$ 涂层热/力学性能的影响
}

\author{
安文然，黄晶琪，卢祥荣，蒋佳宁，邓龙辉，曹学强 \\ (武汉理工大学 硅酸盐建筑材料国家重点实验室, 武汉 430070)
}

摘 要: 大气等离子喷涂制备的 $\mathrm{LaMgAl}_{11} \mathrm{O}_{19}$ ( $\mathrm{LMA}$ )热障涂层无定型相含量较高, 会严重影响涂层服役寿命。通过 900 1600 ${ }^{\circ} \mathrm{C}$ 不同温度热处理 $12 \mathrm{~h}$, 研究晶粒尺寸和孔隙率等微观结构和无定形相含量对 LMA 涂层力学、热物理 以及抗热震性能的影响。结果表明: 喷涂态 LMA 涂层具有 900 和 $1163{ }^{\circ} \mathrm{C}$ 两个结晶温度点。900 ${ }^{\circ} \mathrm{C}$ 热处理后, LMA 涂层中含有较多的无定形相以及最高的孔隙率 $((18.88 \pm 2.15) \%), 1000{ }^{\circ} \mathrm{C}$ 测试时, 具有最低的热扩散系数 $\left(0.53 \mathrm{~mm}^{2} / \mathrm{s}\right)$; 由于重结晶和烧结作用使得无定型相含量和孔隙率降低, $1100 \sim 1400{ }^{\circ} \mathrm{C}$ 之间热处理的涂层具有较高 的硬度 $\left(1100^{\circ} \mathrm{C}\right.$ 时达到最高值 $\left.(12.08 \pm 0.58) \mathrm{GPa}\right) ; 1300{ }^{\circ} \mathrm{C}$ 热处理的涂层中含有大量微米级片状晶, 具有较高的应变 容限以及平均热循环寿命 (588 次); 热处理温度达到 $1500{ }^{\circ} \mathrm{C}$ 时, 由于片状晶平行堆叠, 晶粒厚度迅速增加, 孔隙率 增加, 力学性能显著降低。热震过程中由于热应力的反复作用, 涂层内出现晶粒破碎和裂纹扩展等现象, 导致涂层 最终失效。

关 键 词: $\mathrm{LaMgAl}_{11} \mathrm{O}_{19}$; 热障涂层; 热处理; 热/力学性能

中图分类号: TB306 文献标志码: A

\section{Effect of Heat-treatment Temperature on Thermal and Mechanical Properties of $\mathrm{LaMgAl}_{11} \mathrm{O}_{19}$ Coating}

\begin{abstract}
AN Wenran, HUANG Jingqi, LU Xiangrong, JIANG Jianing, DENG Longhui, CAO Xueqiang
(State Key Laboratory of Silicate Materials for Architectures, Wuhan University of Technology, Wuhan 430070, China)
\end{abstract}

\begin{abstract}
LaMgAl}_{11} \mathrm{O}_{19}$ (LMA) coating prepared by atmospheric plasma spraying has large amounts of amorphous phase, which seriously affects the service life of coating. Effects of microstructure, such as grain size, porosity and amorphous phase content, on mechanical, thermophysical and thermal shock resistance properties of LMA coatings after heat-treatement at $900-1600{ }^{\circ} \mathrm{C}$ for $12 \mathrm{~h}$ were investigated. The results show that the as-sprayed LMA coating possesses two crystallization temperature points, 900 and $1163{ }^{\circ} \mathrm{C}$. After heat-treatement at $900{ }^{\circ} \mathrm{C}$, the lowest thermal diffusivity of $0.53 \mathrm{~mm}^{2} / \mathrm{s}$ was obtained for LMA coating at $1000{ }^{\circ} \mathrm{C}$ due to the large amount of amorphous phase and the highest porosity of $(18.88 \pm 2.15) \%$. LMA coatings, heat-treated at $1100-1400{ }^{\circ} \mathrm{C}$, exhibited higher hardness owing to reduced amorphous content and porosity through recrystallization and sintering with the maximum hardness of (12.08 \pm 0.58$) \mathrm{GPa}$ at $1100{ }^{\circ} \mathrm{C}$. After heat-treatement at $1300{ }^{\circ} \mathrm{C}$, the coating displayed the highest average thermal cycling life (588 times), which was attributed to abundant micron flake crystals with high strain tolerance. When the heat-treatment temperature reached $1500{ }^{\circ} \mathrm{C}$, the grain thickness increased rapidly due to parallel stacking
\end{abstract}

收稿日期：2021-11-22；收到修改稿日期：2022-01-19; 网络出版日期：2022-02-14

基金项目: 国家自然科学基金重点项目(92060201) National Natural Science Foundation of China (92060201)

作者简介: 安文然(1996-), 女, 硕士研究生. E-mail: anwenran1019@126.com AN Wenran (1996-), female, Master candidate. E-mail: anwenran1019@126.com

通信作者：曹学强，教授. E-mail: xcao@whut.edu.cn CAO Xueqiang, professor. E-mail: xcao@whut.edu.cn 
of lamellar crystals, porosity increased and mechanical properties significantly decreased. During the thermal shock, grain breaking and crack propagation occurred in the coating due to the repeated thermal stress, resulting in final failure of the coating.

Key words: $\mathrm{LaMgAl}_{11} \mathrm{O}_{19}$; thermal barrier coating; heat-treatment; thermal and mechanical property

热障涂层(Thermal barrier coatings, TBCs)为燃 气轮机的热端部件提供隔热和防腐蚀保护, 从而提 升了发动机效率及寿命 ${ }^{[1]}$ 。典型的 $\mathrm{TBCs}$ 具有双层 结构: 耐腐蚀、耐氧化的金属黏结层(MCrALY)和隔 热陶瓷层 ${ }^{[2]}$ 。目前, 最常见的为 $6 \% \sim 8 \%$ (质量分数) 的氧化钇部分稳定的氧化锆(YSZ)涂层, 它具有高 熔点、低导热系数以及与金属基体相近的热膨胀系 数等优异的热物理性能 ${ }^{[3]}$, 但只能在 $1200{ }^{\circ} \mathrm{C}$ 以下 稳定工作, 这是其致命的弱点 ${ }^{[4]}$ 。如果长期在 $1200{ }^{\circ} \mathrm{C}$ 以上服役, YSZ 会严重烧结, 杨氏模量增加, 并发生 $\mathrm{t}-\mathrm{ZrO}_{2} \rightarrow \mathrm{m}-\mathrm{ZrO}_{2}$ 的相变且伴随 $4 \% \sim 6 \%$ 的体积膨胀, 这会导致涂层过早剥落 ${ }^{[5]}$ 。为了找到一种可以在高温 下稳定使用的 $\mathrm{TBC}$ 材料, 研究人员付出了大量努力。

$\mathrm{LaMgAl}_{11} \mathrm{O}_{19}(\mathrm{LMA})$ 是一种具有磁铅石结构的 稀土六铝酸盐, 作为热障涂层材料目前被广泛研 究 $^{[6]}$ 。由于磁铅石结构中的尖晶石层被含有较大 $\mathrm{La}^{3+}$ 的镜面层分隔，抑制了晶体沿 $c$ 轴方向生长，因 此微观上呈片状晶形貌。LMA 具有优异的力学和热 物理性能, 如高熔点、较高热膨胀系数、低热导率 和良好的抗烧结性能 ${ }^{[7]}$ 。但是, 作为 TBC 候选材料, LMA 仍有一些问题亟待解决。大气等离子喷涂技术 (APS)所制备的 LMA 涂层含有大量无定型相, 在高 温服役环境中, 无定型相会发生再结晶并伴有较大 的体积收缩 ${ }^{[8-9]}$, 由于受到高温合金基体的限制, LMA 涂层中产生拉伸应力, 使得 LMA 涂层的可靠 性受到影响, 其次晶粒生长过程中 LMA 晶粒大小 的变化对涂层热物理性能也会产生影响。

本工作研究了不同温度热处理的涂层微观结构 和相组成对其热物理性能的影响, 为 LMA 涂层在 1300 1400 ${ }^{\circ} \mathrm{C}$ 下的工作稳定性及其后续在更高温度 $\left(1500 \sim 1600{ }^{\circ} \mathrm{C}\right)$ 下的应用可能性提供必要的实验和 理论支撑。

\section{1 实验方法}

\section{1 样品制备}

采用固相反应法合成 LMA 粉末。将原料 $\mathrm{La}_{2} \mathrm{O}_{3}(99.99 \%) 、 \mathrm{Al}_{2} \mathrm{O}_{3}(99.99 \%)$ 和 $\mathrm{MgO}$ 粉末在 $1000{ }^{\circ} \mathrm{C}$ 下干燥 $24 \mathrm{~h}$, 随后按 $1: 11: 2$ 的摩尔比分散
在去离子水中, 用锆球研磨 $24 \mathrm{~h}$ 得到浆料, 在 $100{ }^{\circ} \mathrm{C}$ 下干燥 $48 \mathrm{~h}$ 后, 经 $1525{ }^{\circ} \mathrm{C}$ 㷽烧 $12 \mathrm{~h}$ 得到 LMA 粉 末。利用喷雾干燥法(SFOC-16)制备 APS 用粉末。 图 1 为 LMA 粉末微观形貌, 粉末呈球状, 粒径分布 在 32 125 $\mu \mathrm{m}$ 范围。

采用 APS (瑞士 Oerlikon Metco, Multicoat)制备 $1.5 \mathrm{~mm}$ 厚的 LMA 涂层。所有石墨基体 $(30 \mathrm{~mm} \times$ $40 \mathrm{~mm} \times 5 \mathrm{~mm})$ 需用砂纸磨平并用喷砂机 (GP-1, $106 \mu \mathrm{m}, 150$ 目砂)预处理来提高基体的粗糙度，从 而增强涂层与基体之间的结合强度。喷涂前利用等 离子火焰将基体预热至 $200{ }^{\circ} \mathrm{C}$ 左右, 减小喷涂过程 中液滴的骤冷温差, 从而有利于液滴铺展、重结晶 和减少热应力等, 具体喷涂参数如表 1 所示。

\section{2 热处理}

将涂层置于 $700{ }^{\circ} \mathrm{C}$ 马弗炉中保温 $10 \mathrm{~h}$, 除去石 墨基体后得到独立的 LMA 涂层。用于热震实验的 LMA 涂层尺寸为 $5 \mathrm{~mm} \times 20 \mathrm{~mm} \times 1.5 \mathrm{~mm}$, 用于其他 性能测试的涂层尺寸为 $10 \mathrm{~mm} \times 10 \mathrm{~mm} \times 1.5 \mathrm{~mm}$ 。将 涂层置于马弗炉中，经过 900、1100、1200、1300、 1400、1500 和 $1600{ }^{\circ} \mathrm{C}$ 热处理 $12 \mathrm{~h}$ 后分别标记为 $\mathrm{L}_{900} 、 \mathrm{~L}_{1100} 、 \mathrm{~L}_{1200} 、 \mathrm{~L}_{1300} 、 \mathrm{~L}_{1400} 、 \mathrm{~L}_{1500}$ 和 $\mathrm{L}_{1600}$, 未热 处理的涂层标记为 $\mathrm{L}_{\mathrm{rt}}$ 。马弗炉在 $1400{ }^{\circ} \mathrm{C}$ 以下按 $5{ }^{\circ} \mathrm{C} / \mathrm{min}$ 的速率升温, $1400{ }^{\circ} \mathrm{C}$ 以上按 $2{ }^{\circ} \mathrm{C} / \mathrm{min}$ 的速 率升温, 降温过程均按 $5{ }^{\circ} \mathrm{C} / \mathrm{min}$ 速率降至 $200{ }^{\circ} \mathrm{C}$, 然后随炉冷却至室温。喷涂态 LMA 涂层具有两个 结晶温度点, 分别位于 900 和 $1163{ }^{\circ} \mathrm{C}$ (如图 2 所示), 1000 和 $1100{ }^{\circ} \mathrm{C}$ 均位于第一结晶结束 $\left(947{ }^{\circ} \mathrm{C}\right)$ 和第二 结晶开始温度 $\left(1106{ }^{\circ} \mathrm{C}\right)$ 之间, 为避免重复工

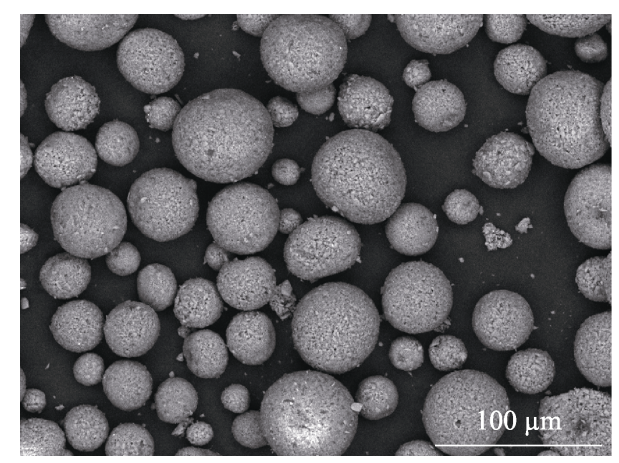

图 1 LMA 粉末 SEM 照片

Fig. 1 SEM image of LMA powder 
表 1 APS 喷涂参数

Table 1 Parameters of air plasma spraying

\begin{tabular}{ccccccc}
\hline Coating & Spray distance $/ \mathrm{mm}$ & Power $/ \mathrm{kW}$ & Current $/ \mathrm{A}$ & Plasma gas Ar $/ \mathrm{H}_{2} / \mathrm{slpm}^{*}$ & Gun velocity $/\left(\mathrm{mm} \cdot \mathrm{s}^{-1}\right)$ & Feeding rate $/\left(\mathrm{g} \cdot \mathrm{min}^{-1}\right)$ \\
\hline LMA & 100 & 42 & 620 & $35 / 12$ & 800 & 35
\end{tabular}

* slpm: Standard liter per minute

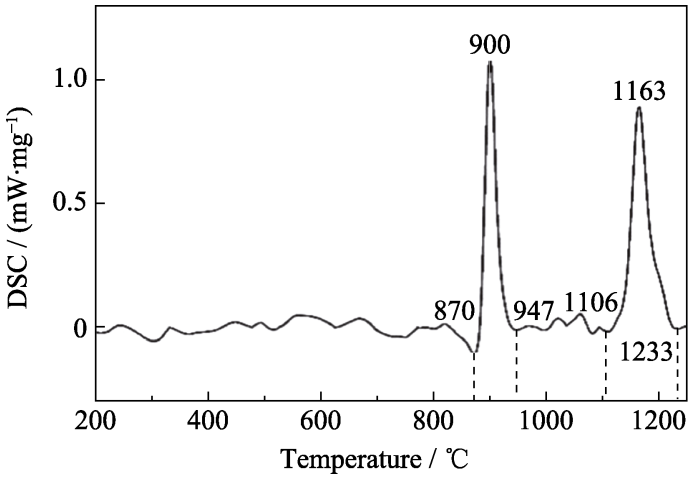

图 2 APS 制备的 LMA 涂层的 DSC 曲线

Fig. 2 DSC curve of LMA coating prepared by APS

作，本研究只选取了 $1100{ }^{\circ} \mathrm{C}$ 进行热处理。通过 $1400{ }^{\circ} \mathrm{C}$ 以上高温短时热处理来探索 LMA 涂层在 1300 1400 ${ }^{\circ} \mathrm{C}$ 长期服役后微观结构及性能的变化, 并为 LMA 涂层在更高温度下的应用提供依据。

\section{3 热震实验}

热震实验在管式炉中进行, 主要过程如下：将 涂层置于 $1200{ }^{\circ} \mathrm{C}$ 的管式炉中保温 $55 \mathrm{~min}$ 后取出, 然后在去离子水中快速冷却至室温。LMA 涂层断裂 则实验结束, 循环次数为其热循环寿命。对 $\mathrm{L}_{\mathrm{rt}} 、 \mathrm{~L}_{900}$ 、 $\mathrm{L}_{1300}$ 和 $\mathrm{L}_{1600}$ 进行了热震实验, 将失效后的涂层分别 标记为 $L_{s r t} 、 L_{s 900} 、 L_{s 1300}$ 和 $L_{s 1600}$ 。每个热处理条件 下取三个样品进行测试，以获得平均热循环寿命。

\section{4 性能测试}

采用日本 Smart Lab 型 X 射线衍射仪(XRD)对 热处理及 CMAS 腐蚀后 LMA 涂层进行物相分析, 测试条件为: $\mathrm{Cu}$ 靶, $2 \theta=10^{\circ} \sim 80^{\circ}$, 步长 $0.02^{\circ}$, 扫描速 度 $4\left(^{\circ}\right) / \mathrm{min}$ 。采用 QUANTA FEG 450 型场发射扫描
电镜 $(\mathrm{SEM})$ 观察涂层断面形貌。将涂层断面镶嵌在 环氧树脂中，用金刚石抛光液进行精细抛光，利用 SEM 观察涂层断面并统计孔隙率, 并利用能谱仪 (EDS)分析元素分布。

采用分析天平(ME204E)测量不同温度热处理 后 LMA 涂层的表观密度(GB/T 25995-2010)。每个 样品称重三次, 以确认涂层的平均密度。

采用德国耐驰仪器公司的 LFA457 型激光导热 仪测试涂层热扩散系数 $(\kappa)$ 。利用 Neumann-Kopp 法 则计算得到涂层比热容 $\left(C_{\mathrm{p}}\right)$, 热导率 $(\lambda)$ 的计算公式 如下 ${ }^{[10]}$ :

$$
\lambda=C_{\mathrm{p}} \cdot \rho \cdot \kappa
$$

其中, $\rho$ 为涂层的表观密度。

\section{2 结果与讨论}

\section{1 物相与微观结构}

不同温度热处理 $12 \mathrm{~h}$ 后 LMA 涂层的 XRD 图谱 如图 3 所示。图 3(a)中 $\mathrm{L}_{\mathrm{RT}}$ 在 $2 \theta=20^{\circ} \sim 36^{\circ}$ 之间的驼 峰属于非晶相, 这是由于大气等离子喷涂过程中熔 融的 LMA 粉末撞击到基体上冷却速度过快, 析晶 被抑制, 从而形成了无定形相 ${ }^{[11-12]}$ 。除了 LMA 外, $\mathrm{L}_{\mathrm{rt}}$ 和 $\mathrm{L}_{900}$ 中还出现了 $\gamma-\mathrm{Al}_{2} \mathrm{O}_{3}$ 和 $\mathrm{La}_{2} \mathrm{O}_{3}$ 相, LMA 粉末在 喷涂过程部分分解为 $\mathrm{MgO} 、 \mathrm{Al}_{2} \mathrm{O}_{3}$ 和 $\mathrm{La}_{2} \mathrm{O}_{3}$, 而 $\mathrm{MgO}$ 饱和蒸气压较高, 在等离子体火焰中选择性蒸发, 因此 XRD 图谱中没有出现 $\mathrm{MgO}$ 的特征峰 ${ }^{[13]}$ 。当热 处理温度高于 $1100{ }^{\circ} \mathrm{C}$ 时，仅检测到 $\mathrm{LMA}$ 和 $\mathrm{LaAlO}_{3}$
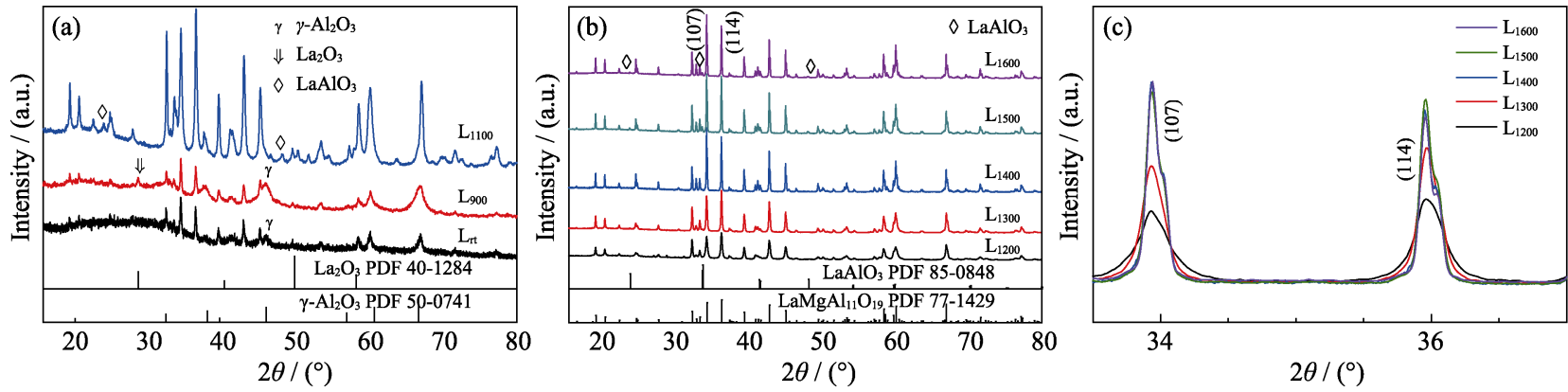

图 3 不同温度热处理后 LMA 涂层的 XRD 图谱

Fig. 3 XRD patterns of LMA coatings after heat-treatement at different temperatures (a) $\mathrm{L}_{\mathrm{rt}}, \mathrm{L}_{900}, \mathrm{~L}_{1100}$; (b) $\mathrm{L}_{1200}, \mathrm{~L}_{1300}, \mathrm{~L}_{1400}, \mathrm{~L}_{1500}$, and $\mathrm{L}_{1600}$; (c) Local magnification XRD patterns at $2 \theta$ between $33.5^{\circ}$ and $37^{\circ}$ of (b) 
的峰, 这是因为 $\mathrm{La}_{2} \mathrm{O}_{3}$ 与 $\gamma-\mathrm{Al}_{2} \mathrm{O}_{3}$ 反应形成了 $\mathrm{LaAlO}_{3}$ 。 随着热处理温度升高, 涂层中非晶相含量减少。当 热处理温度达到 $1200{ }^{\circ} \mathrm{C}$ 时, 非晶相消失, 这是由于 非晶相在 900 和 $1170{ }^{\circ} \mathrm{C}$ 左右发生了重结晶 ${ }^{[8,14-15]}$ 。 当热处理温度高于 $1400{ }^{\circ} \mathrm{C}$ 时, (107)晶面的衍射强 度开始超过(114)晶面(图 3(c)), 表明晶粒开始发生 择优取向生长 ${ }^{[16]}$ 。

图 4 显示了不同温度热处理后 LMA 涂层的断 面形貌。图 4(a)中的层状晶体为未熔融 LMA 晶粒 ${ }^{[17]}$ 。 热处理温度从 $900{ }^{\circ} \mathrm{C}$ 升温到 $1200{ }^{\circ} \mathrm{C}$, 无定型相发 生重结晶过程, 涂层中主要为纳米晶, 图 4(b)中 A 处是非晶相发生重结晶析出的细小晶粒。热处理温 度超过 $1200{ }^{\circ} \mathrm{C}$ 后, 对应晶粒的生长过程。当热处理 温度达到 $1400{ }^{\circ} \mathrm{C}$ 时, 涂层中出现小而薄的片状晶 粒, 这也证明了 $1400{ }^{\circ} \mathrm{C}$ 以上晶粒开始发生择优取 向生长。当热处理温度达到 $1500{ }^{\circ} \mathrm{C}$ 时, 片状晶尺寸 突增, 这是因为为了降低势能, 涂层中小而薄的片 状晶沿着(0001)发生堆叠, 生长成大晶粒。由于晶粒 生长空间有限, 图 $4(\mathrm{~g})$ 显示了晶粒如何被相邻晶粒 限制生长( $\mathrm{B}$ 处) 以及平行晶粒如何堆叠形成大晶粒 (C 处), 这与六铝酸钻晶粒的生长方式相似 ${ }^{[18]}$ 。

利用 Image $\mathrm{J}$ 软件测量了 $\mathrm{L}_{1400} 、 \mathrm{~L}_{1500}$ 和 $\mathrm{L}_{1600}$ 样 品的晶粒厚度和长度, 并利用阿伦尼乌斯方程(2)计 算晶粒厚度和长度方向的生长活化能。

$$
K(T)=A \exp \left(-\frac{E}{R T}\right)
$$

其中, $K(T)$ 为温度为 $T$ 时的晶粒厚度和长度 $(\mathrm{nm}) ; T$ 为热处理温度 $(\mathrm{K}) ; R(8.314 \mathrm{~J} /(\mathrm{mol} \cdot \mathrm{K}))$ 为理想气体常 数; $E$ 为表观活化能 $(\mathrm{J} / \mathrm{mol}) ; A$ 为常数频率因子, 也 称为指数前因子。
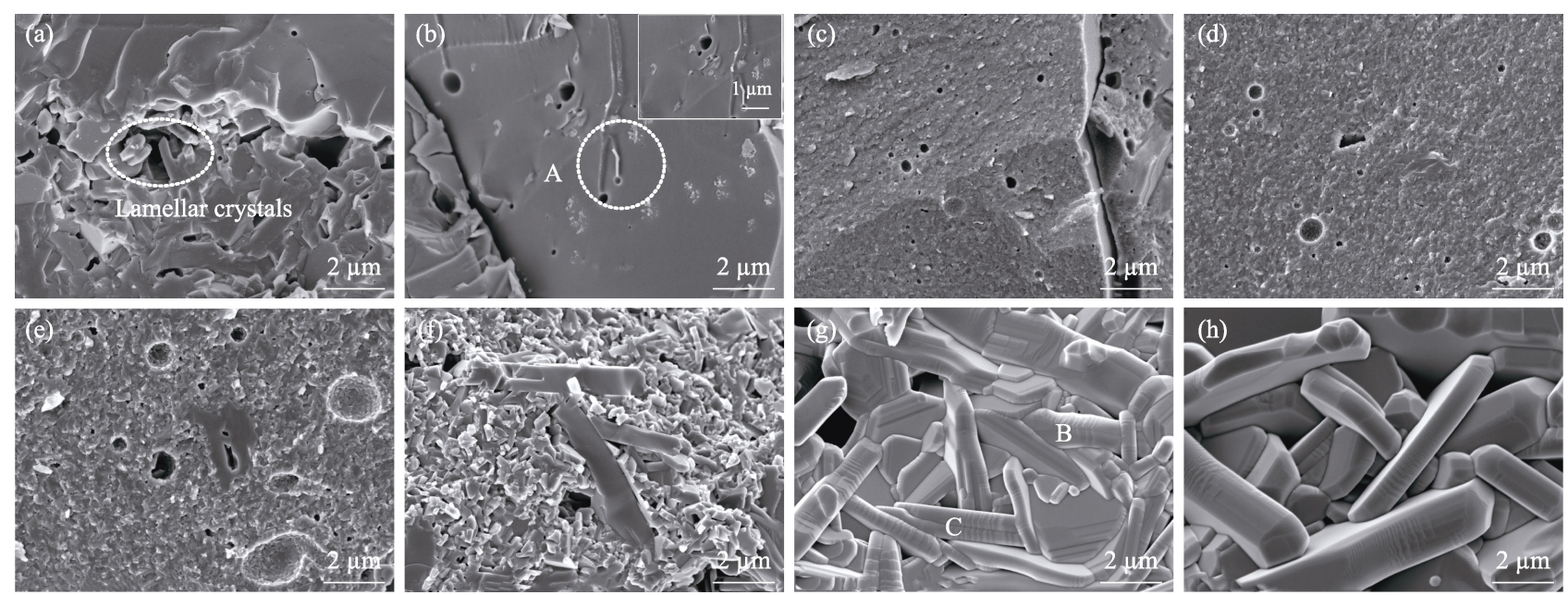

图 4 不同温度热处理后 LMA 涂层的断面 SEM 照片

Fig. 4 Fractured cross-section morphologies of LMA coatings after heat-treatement at different temperatures (a) $\mathrm{L}_{\mathrm{rt}}$; (b) $\mathrm{L}_{900} ;$ (c) $\mathrm{L}_{1100}$; (d) $\mathrm{L}_{1200}$; (e) $\mathrm{L}_{1300}$; (f) $\mathrm{L}_{1400}$; (g) $\mathrm{L}_{1500}$; (h) $\mathrm{L}_{1600}$ 


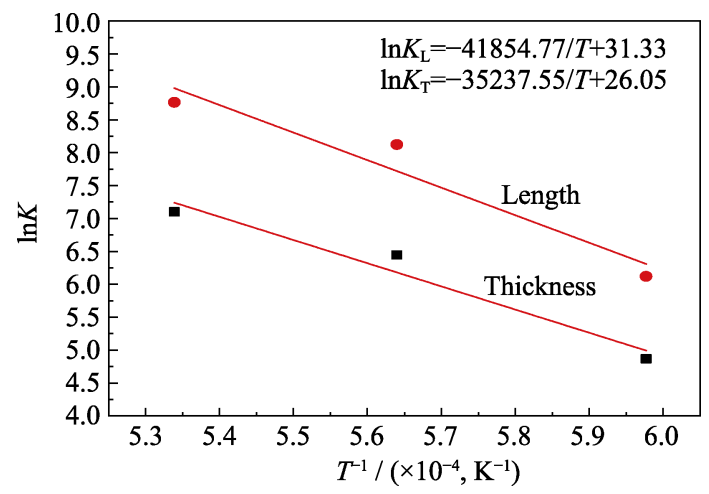

图 5 晶粒的生长活化能

Fig. 5 Activation energy of grain growth

图 9(a)为 $\mathrm{L}_{\mathrm{rt}} 、 \mathrm{~L}_{900} 、 \mathrm{~L}_{1100} 、 \mathrm{~L}_{1200} 、 \mathrm{~L}_{1300} 、 \mathrm{~L}_{1500}$ 和 $\mathrm{L}_{1600}(10 \mathrm{~mm} \times 10 \mathrm{~mm} \times 1.5 \mathrm{~mm})$ 涂层的热扩散系数 曲线, 测试温度范围为 $\mathrm{rt} \sim 1000{ }^{\circ} \mathrm{C}$, 测试间隔为 $200{ }^{\circ} \mathrm{C}$ 。随着温度升高, 涂层热扩散系数逐渐增大且 高于 $\mathrm{L}_{\mathrm{rt}}\left(\mathrm{L}_{900}\right.$ 除外 $) 。 \mathrm{~L}_{900}$ 的热扩散系数最低, 测试温 度为 $1000{ }^{\circ} \mathrm{C}$ 时其热扩散系数仅为 $0.53 \mathrm{~mm}^{2} / \mathrm{s}$, 这 是由于其具有较高的孔隙率和非晶相含量 ${ }^{[20]}$ 。随着
测试温度升高, 各个样品的热扩散系数均降低。这 是因为声子传导是涂层的主要热传导方式，声子在 非晶相中的平均自由程较短。另外，随测试温度升 高声子密度增加, 碰撞加剧, 平均自由程又显著减 小，导致热扩散系数降低 ${ }^{[21]}$ 。利用方程(1)计算涂层 的热导率, 并绘制成图 9(b)。结果表明, 热导率与热 扩散系数具有相同的变化趋势。

\section{3 抗热震性能测试}

对 $\mathrm{L}_{\mathrm{rt}} 、 \mathrm{~L}_{900} 、 \mathrm{~L}_{1300}$ 和 $\mathrm{L}_{1600}$ 进行抗热震试验，热循 环寿命分别为 544、524、588 和 387 次，其中 $\mathrm{L}_{1300}$ 的 热循环寿命最长, 这主要是由于其含有大量的亚微米 级片状晶粒, 从而有利于提高涂层的应变容限 ${ }^{[13,22]}$ 。

对比热震前后样品 XRD 图谱(图 10), 发现热震 后涂层的 XRD 图谱中 $\mathrm{LaAlO}_{3}$ 消失, $\mathrm{La}_{2} \mathrm{O}_{3}$ 和 $\alpha-\mathrm{Al}_{2} \mathrm{O}_{3}$ 出现, 表明 $\mathrm{LaAlO}_{3}$ 在热震过程中发生了分 解。如图 10 所示, $\mathrm{L}_{\mathrm{rt}}$ 和 $\mathrm{L}_{900}$ 含有大量的非晶相, 但 热震后 $\mathrm{L}_{\mathrm{srt}}$ 和 $\mathrm{L}_{\mathrm{s} 900}$ 的结晶度提高, 衍射峰强度增加。 与 $\mathrm{L}_{1300}$ 和 $\mathrm{L}_{1600}$ 相比, $\mathrm{L}_{\mathrm{s} 1300}$ 和 $\mathrm{L}_{\mathrm{s} 1600}$ 的衍射峰强度降 低且衍射峰变宽。
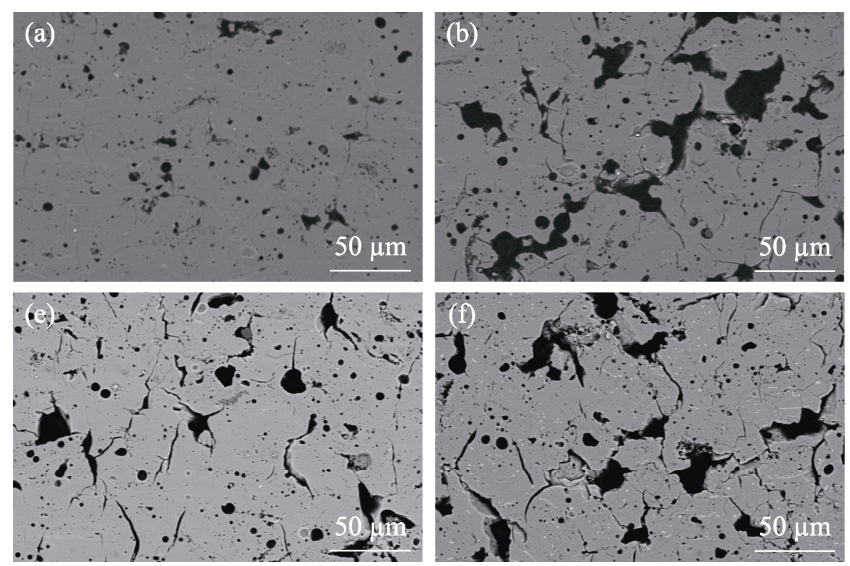
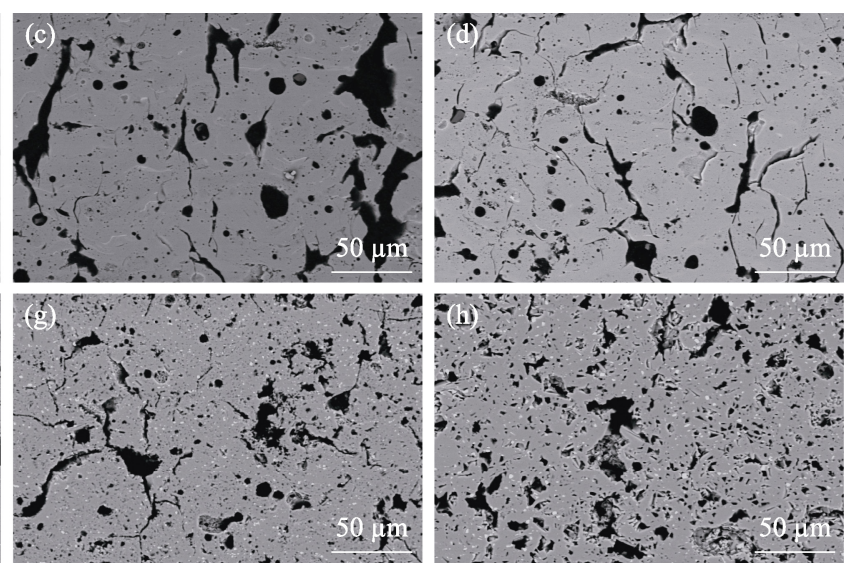

图 6 不同温度热处理后 LMA 涂层截面的 SEM 照片

Fig. 6 SEM images of cross-sectional morphologies of LMA coatings after heat-treatment at different temperatures (a) $\mathrm{L}_{\mathrm{rt}}$; (b) $\mathrm{L}_{900}$; (c) $\mathrm{L}_{1100}$; (d) $\mathrm{L}_{1200}$; (e) $\mathrm{L}_{1300}$; (f) $\mathrm{L}_{1400}$; (g) $\mathrm{L}_{1500}$; (h) $\mathrm{L}_{1600}$

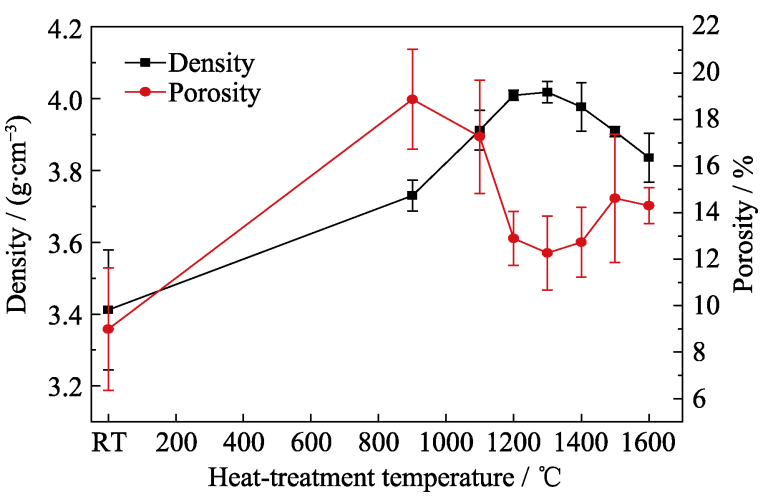

图 7 不同温度热处理后涂层的密度和孔隙率

Fig. 7 Density and porosity of coatings after heat-treatment at different temperatures

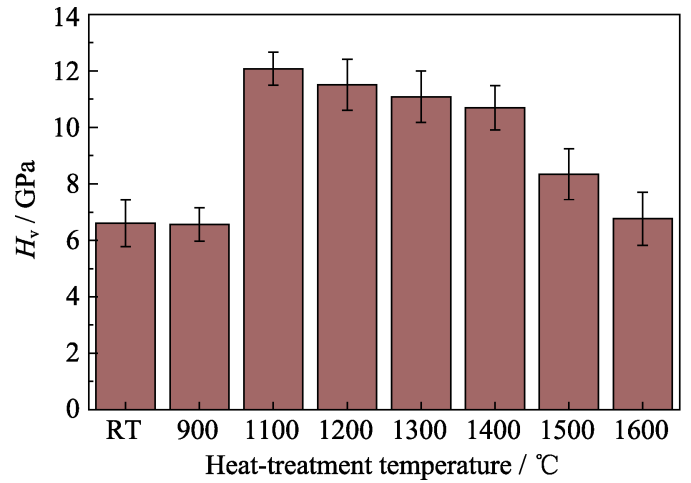

图 8 不同温度热处理后涂层的维氏硬度

Fig. 8 Vickers hardness of coatings after heat-treatment at different temperatures 

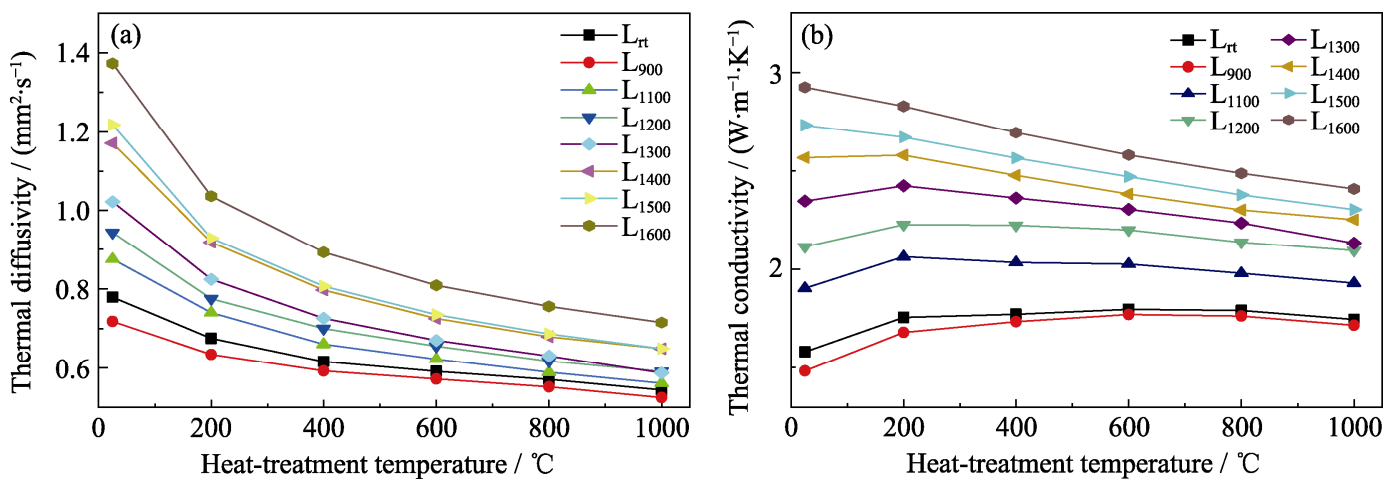

图 9 不同温度热处理后涂层的热物理性能

Fig. 9 Thermophysical properties of coatings after heat-treatment at different temperatures (a) Thermal diffusivity; (b) Thermal conductivity
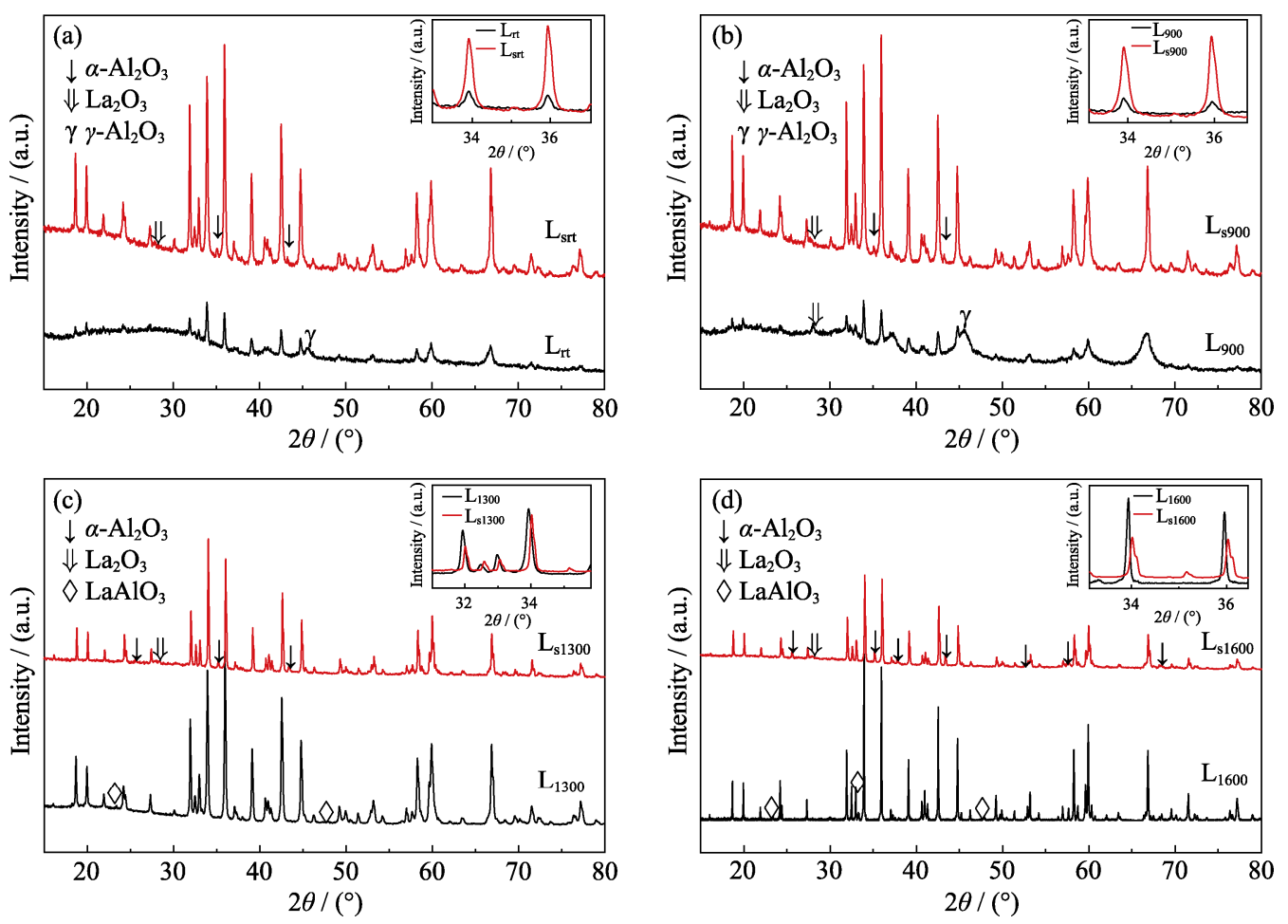

图 10 热震失效前后涂层 XRD 图谱对比

Fig. 10 Comparisons of XRD patterns of coatings before and after thermal shock (a) $\mathrm{L}_{\mathrm{rt}}$ and $\mathrm{L}_{\mathrm{srt}}$; (b) $\mathrm{L}_{900}$ and $\mathrm{L}_{\mathrm{s} 900}$; (c) $\mathrm{L}_{1300}$ and $\mathrm{L}_{\mathrm{s} 1300}$; (d) $\mathrm{L}_{1600}$ and $\mathrm{L}_{\mathrm{s} 1600}$

图 11 显示了 $\mathrm{L}_{\mathrm{rt}} 、 \mathrm{~L}_{900} 、 \mathrm{~L}_{1300}$ 和 $\mathrm{L}_{1600}$ 热震前后 的断面微观形貌, 发现热震失效后涂层中在低倍数 下看似平滑的部分 $(D, E, F, G)$ 是由大量的纳米晶组

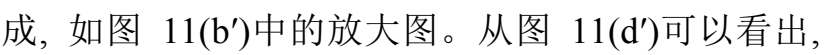
$\mathrm{L}_{1600}$ 中的大尺寸片状晶粒(图 11(d))消失, 这是因为 相对于小晶粒, 大晶粒断裂韧性差, 难以承受水淬 过程中产生的热应力。如图 11( $\left.\mathrm{d}^{\prime}\right)$ 放大图所示, 晶粒 先以片状解离, 然后在热应力作用下断裂形成纳米 晶, 晶粒细化, 这与 $\mathrm{L}_{\mathrm{s} 1600}$ 的 XRD 衍射峰强度降低、 衍射峰变宽的结果一致 ${ }^{[23]}$ 。由 $\mathrm{L}_{\mathrm{srt}} 、 \mathrm{~L}_{\mathrm{s} 900} 、 \mathrm{~L}_{\mathrm{s} 1300}$ 和 $\mathrm{L}_{\mathrm{s} 1600}$ 断面 SEM 形貌可知, 裂纹扩展方式主要为沿 晶扩展: 沿着残余片状晶的晶界(A)和破碎后形成
的纳米晶的晶界(B)扩展, 晶粒尺寸减小, 晶粒界面 与裂纹之间的夹角减小, 这使得裂纹尖端更容易扩 展 ${ }^{[24]}$ 。裂纹还沿着大晶粒的堆叠界面(C)扩展, 因为 堆叠界面结合强度较弱。

表 2 为热震失效前后涂层硬度的对比。由于热 震失效后, $\mathrm{L}_{\mathrm{srt}}$ 与 $\mathrm{L}_{\mathrm{s} 900}$ 结晶度增加, $\mathrm{L}_{\mathrm{s} 1300}$ 与 $\mathrm{L}_{\mathrm{s} 1600}$ 晶 粒破碎, 这使得涂层中含有大量的纳米晶, 维氏硬 度增加 ${ }^{[25]}$ 。

在 $1525{ }^{\circ} \mathrm{C}$ 下对 $\mathrm{L}_{\mathrm{s} 1600}$ 热处理 $12 \mathrm{~h}$ 后(标记为 $\mathrm{L}_{\mathrm{s} 1600-1525}$ ) 发现 $\mathrm{L}_{\mathrm{s} 1600-1525}$ 的晶粒尺寸与 $\mathrm{L}_{1600}$ 相近(图 12(c)), $\mathrm{L}_{\mathrm{s} 1600-1525}$ 的 XRD 图谱中只发现 LMA (图 12(d)), $\mathrm{La}_{2} \mathrm{O}_{3}$ 和 $\alpha-\mathrm{Al}_{2} \mathrm{O}_{3}$ 消失。这是由于经 $1525{ }^{\circ} \mathrm{C}$ 热处理, 

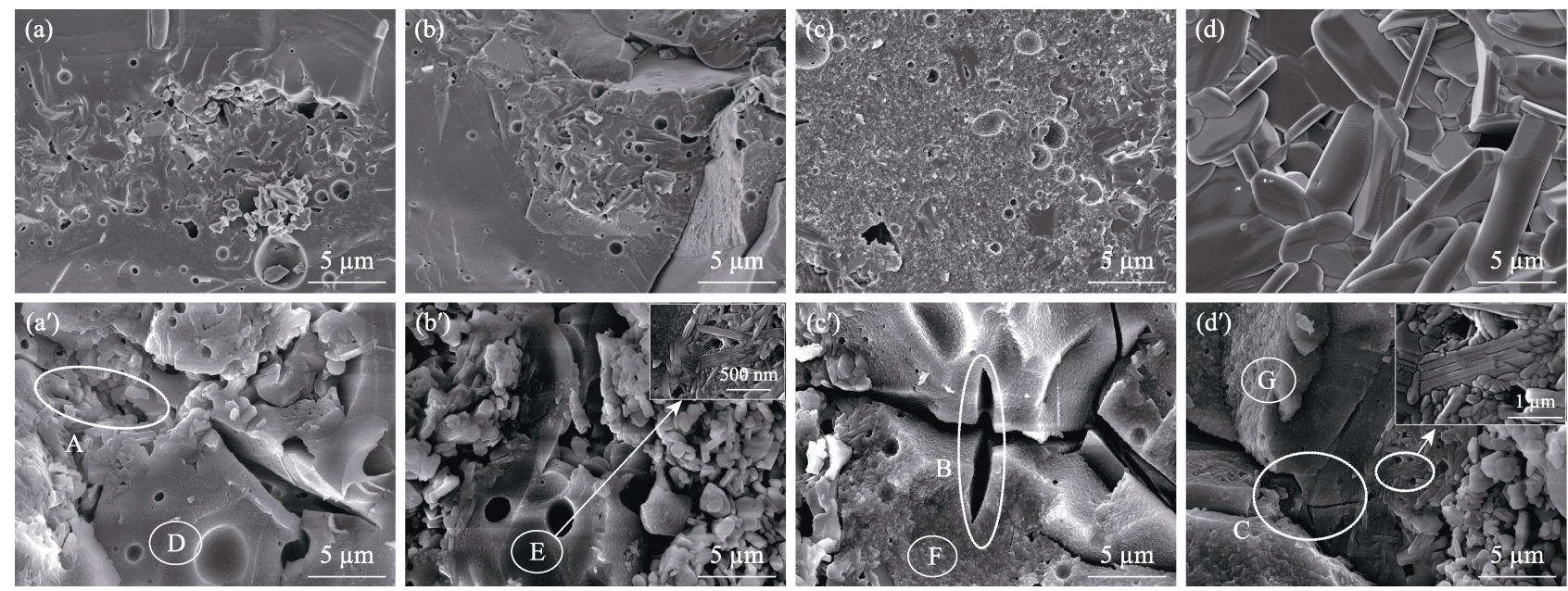

图 11 涂层热震前后涂层断面的微观结构

Fig. 11 Fractured cross-sectional morphologies of coatings before and after thermal shock

(a) $\mathrm{L}_{\mathrm{rt}} ;$ (a') $\mathrm{L}_{\mathrm{srt}} ;$; (b) $\mathrm{L}_{900} ;$ (b') $\mathrm{L}_{\mathrm{s} 900} ;$ (c) $\mathrm{L}_{1300} ;$ (c') $\mathrm{L}_{\mathrm{s} 1300}$; (d) $\mathrm{L}_{1600} ;$ (d') $\mathrm{L}_{\mathrm{s} 1600}$

表 2 涂层热震失效前后硬度对比

Table 2 Vickers hardness of coatings before and after thermal shock

\begin{tabular}{lrrrr}
\hline$H_{\mathrm{V}} / \mathrm{GPa}$ & $\mathrm{L}_{\mathrm{rt}}$ & $\mathrm{L}_{900}$ & $\mathrm{~L}_{1300}$ & $\mathrm{~L}_{1600}$ \\
\hline Before & 6.61 & 6.56 & 11.08 & 6.76 \\
After & 10.12 & 10.89 & 12.07 & 11.01 \\
\hline
\end{tabular}
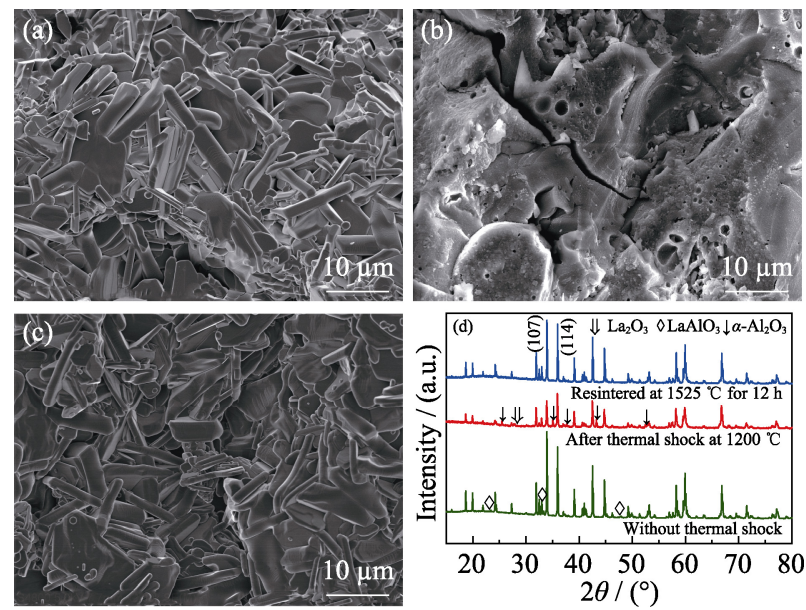

图 $12 \mathrm{~L}_{1600}$ 在不同状态下的 $(\mathrm{a} \sim \mathrm{c})$ 断面微观结构及(d) XRD 图谱

Fig. 12 (a-c) Fractured cross-section morphologies and (d) XRD patterns of $\mathrm{L}_{1600}$ heat-treatment at different conditions (a) $\mathrm{L}_{1600}$; (b) $\mathrm{L}_{\mathrm{s} 1600}$; (c) $\mathrm{L}_{\mathrm{s} 1600-1525}$

$\mathrm{LaAlO}_{3}$ 分解产物与未检测到的 $\mathrm{MgO}$ 反应生成 LMA, 破碎晶粒再次生长成为片状晶。

\section{3 结论}

本工作研究了不同温度热处理后, 涂层相组成 及微观结构对涂层热力学性能、热循环寿命的影响。 热处理温度在 900 1200 ${ }^{\circ} \mathrm{C}$ 之间时, LMA 涂层中无
定型相重结晶, 析出细小晶粒; 热处理温度在 $1200{ }^{\circ} \mathrm{C}$ 以上时, 由于烧结作用, 晶粒逐渐长大, 生成片状 晶; 当热处理温度提高到 $1500{ }^{\circ} \mathrm{C}$ 时, 片状晶发生 堆叠使得晶粒尺寸迅速增加, 孔隙率增加, 从而降 低了涂层硬度。在热震实验过程中, $\mathrm{L}_{1300}$ 中由于含 有大量的微米级片状晶, 具有最高平均热循环寿命 (588 次)。经 $1525{ }^{\circ} \mathrm{C}$ 下热处理 $12 \mathrm{~h}$ 后, $\mathrm{L}_{\mathrm{s} 1600}$ 中破碎 的晶粒与 $\mathrm{LaAlO}_{3}$ 分解产物消失, 重新生成 LMA 片 状晶。

\section{参考文献:}

[1] CAO X Q, VASSEN R, STOEVER D. Ceramic materials for thermal barrier coatings. Journal of the European Ceramic Society, 2004, 24(1): 1-10.

[2] CLARKE D R, OECHSNER M, PADTURE N P. Thermal-barrier coatings for more efficient gas-turbine engines. MRS Bulletin, 2012, 37(10): 891-902.

[3] CLARKE D R, PHILLPOT S R. Thermal barrier coating materials. Materials Today, 2005, 8(6): 22-29.

[4] EVANS A G, CLARKE D R, LEVI C G. The influence of oxides on the performance of advanced gas turbines. Journal of the European Ceramic Society, 2008, 28(7): 1405-1419.

[5] MAO W G, LUO J M, DAI C Y, et al. Effect of heat treatment on deformation and mechanical properties of $8 \mathrm{~mol} \%$ yttria-stabilized zirconia by berkovich nanoindentation. Applied Surface Science, 2015, 338: 92-98.

[6] ZHOU X, HE L M, CAO X Q, et al. $\mathrm{La}_{2}\left(\mathrm{Zr}_{0.7} \mathrm{Ce}_{0.3}\right)_{2} \mathrm{O}_{7}$ thermal barrier coatings prepared by electron beam-physical vapor deposition that are resistant to high temperature attack by molten silicate. Corrosion Science, 2016, 115(16): 143-151.

[7] ZHOU F F, WANG Y, CUI Z Y, et al. Thermal cycling behavior of nanostructured $8 \mathrm{YSZ}, \mathrm{SZ} / 8 \mathrm{YSZ}$ and $8 \mathrm{CSZ} / 8 \mathrm{YSZ}$ thermal barrier coatings fabricated by atmospheric plasma spraying. Ceramics International, 2017, 43(5): 4102-4111.

[8] HUANG L L, MENG H M, TANG J. Crystallization behavior of plasma-sprayed lanthanide magnesium hexaaluminate coatings. International Journal of Minerals, Metallurgy and Materials, 2014, 
21(12): 1247-1253.

[9] LU H R, WANG C A, ZHANG C G, et al. Thermo-physical properties of rare-earth hexaaluminates $\mathrm{LnMgAl}_{11} \mathrm{O}_{19}$ ( $\mathrm{Ln}$ : $\mathrm{La}$, Pr, $\mathrm{Nd}, \mathrm{Sm}, \mathrm{Eu}$ and $\mathrm{Gd}$ ) magnetoplumbite for advanced thermal barrier coatings. Journal of the European Ceramic Society, 2015, 35(4): 1297-1306.

[10] KINGERY W D, MCQUARRIE M C. Thermal conductivity: i, concepts of measurement and factors affecting thermal conductivity of ceramic materials. Journal of the American Ceramic Society, 1954, 37(2): 67-72.

[11] LIU H Z, OUYANG J H, LIU Z G, et al. Microstructure, thermal shock resistance and thermal emissivity of plasma sprayed $\mathrm{LaMAl}_{11} \mathrm{O}_{19}(\mathrm{M}=\mathrm{Mg}, \mathrm{Fe})$ coatings for metallic thermal protection systems. Applied Surface Science, 2013, 217: 52-59.

[12] CAO X Q, ZHANG Y F, ZHANG J F, et al. Failure of the plasma-sprayed coating of lanthanum hexaluminate. Journal of the European Ceramic Society, 2008, 28(10): 1979-1986.

[13] SUN J B, WANG J S, ZHOU X, et al. Thermal cycling behavior of the plasma-sprayed coating of lanthanum hexaaluminate. Journal of the European Ceramic Society, 2018, 38(4): 1919-1929.

[14] ZENG J Y, SUN J B, LIANG P P, et al. Heat-treated lanthanum magnesium hexaaluminate coatings exposed to molten calciummagnesium-alumino-silicate. Ceramics International, 2019, 45(9): 11723-11733.

[15] SUN J B, WANG J S, DONG S J, et al. Effect of heat treatment on microstructure and property of plasma-sprayed lanthanum hexaaluminate coating. Journal of Alloys and Compounds, 2018, 739: 856-865.

[16] CHAO C Y, REN Z H, ZHU Y H, et al. Self-templated synthesis of single-crystal and single-domain ferroelectric nanoplates. Angewandte Chemie International Edition, 2012, 51(37): 9283-9287.

[17] SUN J B, WANG J S, ZHOU X, et al. Microstructure and thermal cycling behavior of plasma-sprayed $\mathrm{LaMgAl}_{11} \mathrm{O}_{19}$ coatings. Ceramics International, 2018, 44(5): 5572-5580.

[18] DOMINGUEZ C, CHEVALIER J, TORRECILlAS R, et al. Microstructure development in calcium hexaluminate. Journal of the European Ceramic Society, 2001, 21(3): 381-387.

[19] SUN X M, DU L Z, LAN H, et al. Study on thermal shock behavior of YSZ abradable sealing coating prepared by mixed solution precursor plasma spraying. Surface \& Coatings Technology, 2020, 397: 126045.

[20] ZHOU X, SONG W J, YUAN J Y, et al. Thermophysical properties and cyclic lifetime of plasma sprayed $\mathrm{SrAl}_{12} \mathrm{O}_{19}$ for thermal barrier coating applications. Journal of the American Ceramic Society, 2020, 103(10): 5599-5611.

[21] TARASI F, MEDRAJ M, DOLATABADI A, et al. Hightemperature performance of alumina-zirconia composite coatings containing amorphous phases. Advanced Functional Materials, 2011, 21(21): 4143-4151.

[22] CHEN X L, ZHANG Y F, ZHONG X H, et al. Thermal cycling behaviors of the plasma sprayed thermal barrier coatings of hexaluminates with magnetoplumbite structure. Journal of the European Ceramic Society, 2010, 30(7): 1649-1657.

[23] 杨晓洁, 常雪婷, 范润华. 快速多重旋转碾压诱导 Ti-6Al-4V 表面纳米晶及性能研究. 表面技术, 2021, 50(5): 177-183.

[24] ARAI Y, INOUE R, KAKISAWA H. Anisotropic crack propagation behavior for the silicon-bond coat layer in a multilayer coated system. International Journal of Applied Ceramic Technology, 2021, 18(3): 947-956.

[25] SONG X M, SUHONEN T, SUN C, et al. Microstructures, microhardness, and crystallization behaviors of amorphous $\mathrm{Al}_{2} \mathrm{O}_{3^{-}}$ YSZ coatings prepared by air plasma spraying. Surface Review and Letters, 2015, 22(4): 1550047. 